\title{
Quantification of Fairness Perception by Including Other- Regarding Concerns Using a Relativistic Fairness-Equity Model
}

\author{
Nicoladie D. Tam \\ Department of Biological Sciences \\ University of North Texas, Denton, TX 76023, USA \\ nicoladie.tam@unt.edu
}

\begin{abstract}
Using a relativistic fairness-equity model, this study quantifies the fairness perception by a stimulus-response function. Fairness often requires the comparison between two parties. The relativistic model takes into the account of the relativity in the comparison, depending on whether the comparison is based on a self-centered or an other-centered frame of reference. Using the Ultimatum Game (UG) experimental paradigm, fairness perception is quantified by the fairness stimulus-response function in human subjects, where the $y$-intercept represents the baseline fairness and the slope represents the fairness sensitivity. The results show that fairness perception is proportional to the offer-ratio between the proposer and the responder using the self-centered frame of reference, with one exception. The exception is that, at absolute equity (equal share between the two parties), the subjects reported the offer as the most fair, even more fair than the most hyper-equitable offers. This suggests that the subjects switch the frame of reference from a self-centered frame of reference to an other-centered frame of reference. That is, by switching from a local (subjective) frame of reference to a global (objective) frame of reference, the optimization for fairness for both parties can be achieved, resolving the relativistic dilemma that fairness for one person is unfairness for another person. This shows that the relativistic model can describe how fairness perception can be biased relative to the two parties, using a self-centered and an other-centered frame of reference.
\end{abstract}

Keywords: Fairness bias; equity; egalitarianism, monetary gain; ultimatum game; decision.

\section{INTRODUCTION}

Fairness is one of the high-level cognitive concepts used to compare and contrast the difference in how we treat others, and how we want to be treated by others. It can affect the behaviors in social interactions depending on how one perceives the treatment as fair or not. It is not only used by human, but also by primates [1] to compare and contrast the unequal treatments. Thus, it is important to determine how fairness is computed quantitatively, so that the biases in fairness perception can be assessed.

Fairness has been studied extensively in relation to social interactions [2-4], especially in relation to the neurobiology of brain functions using brain-imaging technologies [5-10]. It has also been studied in relation to economic transactions [11-15], and distributive justice [16-18]. 


\section{Literature Review of the Computational Models of Fairness Assessment}

There are many different computational models of fairness [19-26] [27], which are mostly based on economic game theories $[13,14,16,17,28]$. Most of these economic fairness models are based on the inequity aversion model [14], in which equity is used as a measure for the assessment of fairness. Although most societies often consider social equality as a measure of fairness, this assessment is often not sufficient to account for the biases in fairness perception. That is, if equity is used as a measure in the comparison, then equality is usually considered as fair; otherwise, inequality is considered as unfair. Yet, fairness and equality are not necessarily equivalent. That is, what is fair may not be equal; what is equal may not be fair. For instance, a big person may eat more food than a small person may, so it is not equal but fair. So there exists a paradox that equity does not necessarily imply fairness; nor does fairness imply equity. Thus, an inequity aversion model does not account for the perception of fairness adequately, because social interactions are not always driven by maximizing gains using mutual-max optimization [13] to achieve equity, but by the relativity of the comparison between oneself and the other person, as proposed in our relativistic fairness-equity model $[29,30]$.

\section{The Relativistic Fairness-Equity Model}

In order to account for the relative perception of fairness, a relativistic fairness-equity model $[29,30]$ was introduced to compare the relative difference between oneself and the other person in the assessment of fairness. It differs from the other fairness models, in which it takes into the account of relativity between self and others. The relativity is determined by whether the frame of reference used in the comparison is based on self or others. For instance, if the quantity is a desirable quantity (such as any valuables), then the perception of fairness is dependent on who has more in the comparison. That is, if others have more than self, then it is often considered as unfair to self - using the self-centered frame of reference (assuming everything else used in the comparison are equal for both parties). At the same time, it is also hyper-fair to others, if the other-centered frame of reference is used. Thus, the result of the comparison is often opposite to each other, if the reference is switched from a self-centered to an other-centered frame of reference. More importantly, if the comparison is inclusive of both self and others, using a global frame of reference, instead of a local frame of reference, then the perception of fairness can change significantly. Therefore, fairness is highly dependent on which frame of reference is used in computing the difference or the disparity between the parties.

\section{Hypothesis}

Based on the relativistic fairness-equity model, the hypothesis is that fairness perception is proportional to the disparity between self and others in the comparison, depending on the frame of reference. If the frame of reference is switched from a self-centered frame of reference to an other-centered frame of reference, then humans can evaluate fairness objectively. The decision is often based on optimizing the gain for oneself, using the selfcentered frame of reference to include self-regarding concerns. However, in most social interactions, such decision could also include other-regarding concerns. This can be done by taking into account the other-centered frame of reference to optimize the gain for other individuals, if such inclusiveness does not conflict with the self-regarding concerns. That is, if optimizing the gain for oneself does not produce a conflict with the optimization for the gain for others, then humans often opt for optimizing the gain for both parties.

Since optimizing the gain for oneself often leads to fairness for oneself, but unfairness for others, according to our relativistic fairness-equity model, it creates a conflict so that 
optimizing fairness for both is often not possible. This is because conflict arises when a person has to choose between two options, but it is only possible to choose one but not the other. In a conflict, choosing one of the options automatically nullifies the option for the other. Thus, if a person wants to optimize fairness for both parties, then maximizing gain for oneself would automatically minimize gain (or maximize loss) for the other person, due to the relativity of fairness assessment for oneself vs. others. However, at absolute equity, it is possible to optimize the gain for both, which results in fairness for both parties without diminishing the gain for oneself or the other.

Thus, our hypothesis will predict that fairness perception is assessed by using a self-centered frame of reference while considering an other-centered frame of reference; and the fairness consideration will include the other-centered frame of reference, only if it does not conflict with the self-regarding concerns (i.e., being fair to oneself). The condition to achieve fairness for both is possible at absolute equity. Thus, humans will include the other-regarding considerations at equity, and perceive this even-share equity condition as the fairest of all.

\section{Quantification of Fairness}

In order to assess how fairness perception can be biased, the stimulus-response function is used to quantify fairness. Using the quantifiable stimulus-response function, the relativistic fairness-equity model can determine how fairness is biased by the shifting of the stimulusresponse curve graphically relative to the selected frame of reference [30]. By quantifying how fairness is biased in the computation, we can determine what factors could have contributed to these biases, and how they can affect the social interactions.

\section{Ultimatum Game Experimental Paradigm}

In assessing the relationship between fairness and decision, Ultimatum Game (UG) is one of the most widely used experimental paradigms in behavioral economics, social science, psychology, neuropsychology, and mathematical psychology [11, 20, 24, 31-33]. It is a simple split-themoney game to ask human subjects to accept or reject the monetary offer, which can be dependent on how the subjects consider the offer as fair or not. The rule of the game is that a sum of money (such as \$10) is split between two persons - a proposer and a responder. The proposer offers a share of the money (such as \$3) to the responder, while keeping another share for himself/herself (such as \$7). The responder is asked to accept or reject the offer. If the responder accepts the offer, both keep their share of money. If the responder rejects, both lose the money.

\section{Objectives}

The objective of this study is to determine whether fairness perception can be quantified by the stimulus-response function, according to the relativistic fairness-equity model, using the UG experimental paradigm. That is, we will address the hypothesis that the fairness perception is proportional to the disparity between two persons, if it uses a self-centered frame of reference. However, when the frame of reference is switched to an other-centered frame of reference, then the fairness perception would be altered, deviating from the original selfcentered perception. If such scenario occurs, then the stimulus-response function can be used to quantify whether the other-regarding consideration would alter the fairness perception from the proportionality relationship relative to the self-centered frame of reference. That is, if fairness bias exists, then the stimulus-response function would be shifted/skewed relative to the equity (disparity) measure graphically. Thus, the fairness bias can be quantified by the amount of fairness deviated from the predicted fairness in stimulus-response function. We want to determine whether the fairness perception at equity will deviate significantly from the self-centered frame of reference in accessing fairness. If so, then fairness is assessed by taking 
into account of considerations other than the self-regarding considerations, according to the relativistic fairness-equity model.

\section{Experimental Methodology}

\section{METHODS}

We recruited healthy human subjects to participate in this study voluntarily. The subjects were asked to play the ultimatum game as a responder. An amount of money is proposed to the human subject to accept or reject on the computer screen by the proposer. The computer is used as the proposer, because we did not want to bias the subject's fairness perception, if human intervention were used as the proposer; especially since it has been shown that knowledge of human vs. computer proposer could bias the fairness perception in UG [8, 34]. No confederates or any forms of deception were used to alter the subject's perception, so that the experimental conditions were presented as neutral as possible, without experimentally manipulating the subject's fairness perception. The experiments were self-paced by the subjects without having any time pressure, external constraints or manipulations.

Each experiment was performed with nine randomized offers proposed to the responder, which ranged from $\$ 1$ to $\$ 9$. All experiments were one-shot trial experiments, i.e., the offers were presented once, without repeating any offer twice. The offer-ratios were presented in random order to the responder, which ranged from a stingy $\$ 1: \$ 9$ offer to a generous $\$ 9: \$ 1$ offer. When the responder accepted (or rejected) the offer, the subject was asked to rate how fair the offer was in a scale of +5 to -5 Likert scale [35]. We also asked the subject to rate other attributes (such as, how important fairness is; how important money is; how important winning is; whether they won the trial; in addition to other emotions: happy, angry, sad and jealous using the same rating scale). The extra questions served, in part, as distracters to reduce the likelihood that the subject may skew the responses if the objectives of the experiment were known. The relationships between emotions and the monetary gain with respect to fairness had been reported elsewhere [36-38].

By design, we used the same pseudo-random sequence of monetary offers to ensure consistency in the experimental conditions across all subjects. To identify whether the subject's perception had changed in the course of the experiment, we also recorded their emotional state at the beginning and end of experiment. By design, we specifically measured the subject's self-reported ratings, because the self-reported measures encapsulate the subjective perception, which are filtered through their own subjective biases. Thus, if bias existed in their fairness perception, that bias would also be reported as a skewed response in the self-reported rating of fairness. Thus, the bias will be revealed in the subsequent analysis. This study was approved by the University Institutional Review Board. Informed consent was provided to the subjects prior to the beginning of the experiment.

\section{RESULTS}

This study included a total of 425 human subjects (age ranging from 18 to 80, median $=21$; mean $=22.3 ; \mathrm{SD}=4.7 ; 275$ female, 150 male). Fig. 1 shows the fairness stimulus-response graph of the entire sampled population with respect to the offer-ratios. The self-reported fairness ratings are plotted in the y-axis, while the offer-ratios (which is sorted from the randomized offer-ratios) are plotted in the $\mathrm{x}$-axis. 


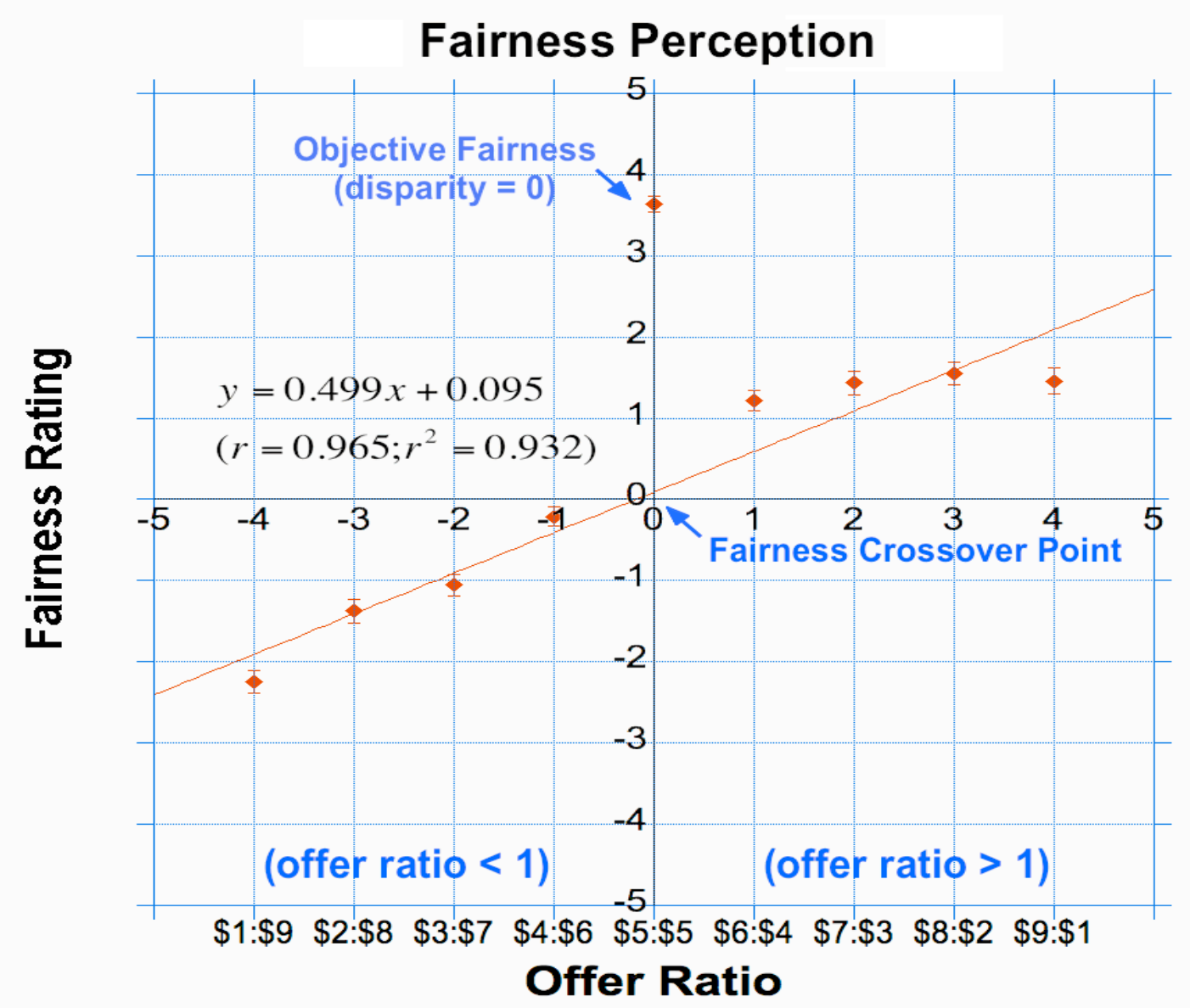

Figure 1: Stimulus-response graph of fairness vs. offer-ratios for all trials (i.e., including both acceptance and rejection trials). Curve-fitting is done by regression for all data points (excluding singularity-point at offer-ratio $=0$ ). It shows a linear proportionality relationship between fairness rating and offer-ratio. Note that the fairness rating at absolute equity (offer-ratio $=1$ ) is the highest, even higher than the hyper-equitable offers (offer-ratio $>1$ ). The error bar represents standard error of mean (SEM).

\section{Proportionality in Fairness Perception to Equity-Ratio}

Fig. 1 displays the regression line $(\mathrm{r}=0.963 ; \mathrm{r} 2=0.932)$ fitted through the data. It shows a direct proportionality relationship between the fairness rating and the offer-ratios, indicating that the more equitable the offer is, the more fair the subject perceives the offer. This shows fairness is proportional to the equity-ratio between the proposer and the responder ( $y=$ $0.499 \mathrm{x}+0.095)$, as predicted by the relativistic fairness-equity model.

\section{Self-Centered Frame of Reference in Evaluating Fairness}

Note that the fairness crossover point (based on the regression line) is precisely located at absolute equity (offer-ratio $=\$ 5: \$ 5$ ) with a fairness rating $=0$ (neutral fairness). That is, at even-split, there is no disparity between the two parties, so fairness perception should be neutral - i.e., neither fair nor unfair. This is consistent with the self-centered frame of reference in determining fairness according to the disparity between self and others. That is, if the offer-ratio is less than one (stingy offers with offer-ratio $<1$ ), it is inequitable, so it would be perceived as unfair. Indeed, the subjects rated it as unfair, which is consistent with the selfcentered fairness-equity hypothesis. On the other hand, if the offer-ratio is greater than one (generous offers with offer-ratio $>1$ ), it is hyper-equitable, so it would be perceived as hyperfair. Indeed, the subjects rated it as hyper-fair, which is also consistent with the self-centered fairness-equity hypothesis. 


\section{Other-Centered Frame of Reference in Evaluating Fairness at Absolute Equity}

Most interesting, by interpolating the fairness stimulus-response function using the regression line $(y=0.499 x+0.095)$, the predicted fairness rating is zero (y-intercept $=0.095 \approx 0)$ at absolute equity (offer-ratio $=\$ 5: \$ 5$ ), passing through the origins of both $\mathrm{x}$-axis and y-axis exactly. This means that the fairness rating would have been "neutral" in the self-reported rating - i.e. neither fair nor unfair - had the subjects used the self-centered frame of reference in assessing fairness. Indeed, the regression line does precisely pass through the axes-origin.

Contrary to this self-centered assumption, the data show that the self-reported rating of fairness at absolute equity is 3.7 point (instead of 0 ) in the 10 -point scale of +5 to -5 . In fact, it is two times more fair than the most hyper-equitable/hyper-fair (generous) offer (which is rated at 1.6 for offer-ratio of $\$ 9: \$ 1$ ). Thus, it suggests that there is a switch from the subjective self-centered frame of reference to an objective other-centered frame of reference at absolute equity (offer-ratio of $\$ 5: \$ 5$ ). That is, instead of perceiving the fairness as neutral when there is no disparity between the two parties at absolute equity, the subjects perceived the offer as extremely fair, even more fair than the most generous offers.

\section{Switching from a Subjective Frame of Reference to an Objective Frame of Reference at Absolute Equity}

This singularity point in the proportional fairness-equity curve is a significant deviation from the self-centered frame of reference in assessing fairness. To account for this deviation, the subjects were most likely to have included the other-centered frame of reference in the evaluating of fairness, such that it is simultaneously fair to oneself and fair to the other party, regardless of which frame of reference is used. That is, the subjects were likely to optimize the fairness for both parties, but it is not possible to be both fair to self and fair to others if the offers are inequitable. That is, for inequitable offers, it is unfair to oneself, but hyper-fair to the other person. For hyper-equitable offers, it is hyper-fair to oneself, but unfair to the other person. This creates a dilemma in optimizing fairness for both.

However, this dilemma is resolved at absolute equity (offer-ratio $=1$ ). It can be fair to both parties. Thus, the fairness perception is doubly fair when it is fair to oneself and fair to the other person simultaneously. This also suggests that the perception of fairness for equality is a result of an internal optimization process for resolving fairness for both parties, using a global frame of reference instead of a local frame of reference.

\section{Relativistic Fairness Comparison by Optimizing Fairness for Both Parties}

The deviation of the singularity point at absolute equity can be accounted for by the relativistic fairness-equity model, which captured this phenomenon by switching from the self-centered frame of reference to the other-centered frame of reference in evaluating fairness. If a selfcentered frame of reference was used, the interpolation of the regression line clearly predicts that the human subjects could have perceived the fairness level as neutral (fairness rating $=0$ ) for the offer-ratio of $\$ 5: \$ 5$. Instead, the subjects rated it as 3.7, more than twice as high as the fairest rating for hyper-equitable/hyper-fair generous offers. Thus, the subjects most likely switched from the self-regarding concerns to the other-regarding concerns in assessing fairness. At equality (absolute equity), it would be fair for both persons at the same time. Thus, it is no longer hyper-fair for one person, while unfair for another person at the same time. Equal sharing resolves the optimization dilemma by being inclusive of both self and 
others, rather than being self-centered. Thus, egalitarianism is a phenomenon that is more likely to be a consequence of the internal optimization process to optimize fairness for both parties than a behavior that is imposed externally by social norms.

\section{DISCUSSIONS}

The analysis shows that the fairness rating level is consistent with the hypothesis that the fairness perception is proportional to the disparity in the offer-ratio, which is consistent with other fairness studies $[2,5,6,9,14,16]$. The more equitable the offers were, the fairer the selfreported ratings were, but with one exception. The exception that deviated from this proportionality relationship is at absolute equity (equal share). At equality, the fairness perception is rated the highest, indicating that the fairness perception is considered as the most fair.

The analysis validates the relativistic fairness-equity model by its ability to account for the singularity point at the absolute equity point as the most fair, even more fair than any of the other hyper-equitable/hyper-generous offers. This shows that the inequity aversion model does not completely account for this anomaly, if a single self-centered frame of reference is used in evaluating fairness. This suggests that humans most likely consider both frames of reference to evaluate fairness. By including both self-centered and other-centered frames of reference, it can resolve the dilemma that exists when maximizing one's gain would imply minimizing another person's gain. At absolute equity (equality), the dilemma is resolved by optimizing fairness for both parties without any conflicts, which is reflected in the highest rating of fairness. This objective fairness perception is more fair than the subjective selfcentered perspective of fairness.

The analysis suggests that human preference for absolute equity is not necessarily driven by the external social norm in egalitarianism, but by considering both self-centered and othercentered frames of reference in evaluating fairness. That is, at absolute equity (offer-ratio $=1$ ), the fairness perception deviates from the self-centered frame of reference (that only considers self-regarding concerns, but also considers other-regarding concerns as well). Thus, fairness is not determined by the monetary gain (or disparity), but by the consideration of others.

Other UG studies of emotions also showed a similar singularity point that deviates from the proportionality relationship to offer-ratios (or monetary gain). Specifically, human subjects reported happier at absolute equity than other offer-ratios [36], regardless of whether they accepted or rejected the offers [37]. Similarly, human subjects also reported less angry at absolute equity than other offer-ratios [38]. Thus, these results are consistent with the interpretation that humans switch the frame of reference from a self-centered one to an othercentered one at absolute equity. They are happier and less angry when the offer is equal to both parties than any other offers. This suggests that humans include other-regarding concerns not only in their relativistic perception of fairness, but also in their perception of emotions.

This shows that egalitarianism is more likely to be an internally generated optimization process in which the human subjects included both self-regarding and other-regarding concerns in the evaluation of fairness, without necessarily using an external-imposed social norm to guide egalitarian behaviors. The companion paper [39] will address whether the same proportionality stimulus-response relationship is preserved depending on the decision to accept or reject the offers. The companion paper will also provide evidence that not only is the proportionality relationship preserved, but also the egalitarian perception of fairness is preserved as the highest, even when the subjects decided to reject the offers. 


\section{CONCLUSION}

This study shows that fairness perception can be quantified by the relativistic fairness-equity model to account for the switching from a self-centered frame of reference to an othercentered frame of reference at absolute equity. Using the UG paradigm, it shows fairness perception is proportional to the disparity between oneself and the other person, except at absolute equity. This subjective fairness perception is based on a self-centered frame of reference, except at absolute equity (equal share). At equality, the frame of reference is switched to include both the self-centered and the other-centered frame of reference. The singularity point at absolute equity is the fairest of all, which deviates from the proportionality relationship that is based on the self-centered frame of reference. Thus, it suggests that humans use a self-centered frame of reference in assessing fairness, while including the othercentered frames of reference if optimizing the fairness for both is feasible (which occurs at absolute equity). Thus, egalitarian behaviors can be generated internally by optimizing fairness for both parties, without relying on the external social norms for guidance. The experimental data validated the theoretical prediction that uses relativity in fairness assessment as proposed by the relativistic fairness-equity model.

\section{ACKNOWLEDGEMENT}

I thank Ms. Krista Smith for providing helpful suggestions and proofreading the manuscript.

\section{References}

1. Brosnan, S.F. and F.B. De Waal, Monkeys reject unequal pay. Nature, 2003. 425(6955): p. $297-299$.

2. Fehr, E. and S. Gächter, Altruistic punishment in humans. Nature, 2002. 415(6868): p. 137-140.

3. Pillutla, M.M. and J.K. Murnighan, Unfairness, Anger, and Spite: Emotional Rejections of Ultimatum Offers. Org Behav Human Decis Proc, 1996. 68(3): p. 208-224.

4. Seip, E.C., W.W. van Dijk, and M. Rotteveel, On hotheads and Dirty Harries: the primacy of anger in altruistic punishment. Ann N Y Acad Sci, 2009. 1167: p. 190-196.

5. Güroğlu, B., et al., Unfair? It depends: neural correlates of fairness in social context. Soc Cogn Affect Neurosci, 2010. 5(4): p. 414-423.

6. Güroğlu, B., W. van den Bos, and E.A. Crone, Fairness considerations: increasing understanding of intentionality during adolescence. J Exp Child Psychol, 2009. 104(4): p. 398-409.

7. Rilling, J.K., B. King-Casas, and A.G. Sanfey, The neurobiology of social decision-making. Curr Opin Neurobiol, 2008. 18(2): p. 159-165.

8. Sanfey, A.G., et al., The neural basis of economic decision-making in the Ultimatum Game. Science, 2003. 300(5626): p. 1755-1758.

9. Tabibnia, G., A.B. Satpute, and M.D. Lieberman, The sunny side of fairness: preference for fairness activates reward circuitry (and disregarding unfairness activates self-control circuitry). Psychol Sci, 2008. 19(4): p. 339-347.

10. Takagishi, H., et al., Neural correlates of the rejection of unfair offers in the impunity game. Neuro Endocrinol Lett, 2009. 30(4): p. 496-500.

11. Güth, W., R. Schmittberger, and B. Schwarze, An experimental analysis of ultimatum bargaining. J Econ Behav Organization, 1982. 3(4): p. 367-388. 
12. Ochs, J. and A.E. Roth, An experimental study of sequential bargaining. Am Econ Review, 1989. 79(3): $p$. 355-384.

13. Rabin, M., Incorporating fairness into game theory and economics. Am Econ Review, 1993. 83(5): p. 1281-1302.

14. Fehr, E. and K.M. Schmidt, A theory of fairness, competition, and cooperation. Quarterly J Econ, 1999. 114: p. 817-868.

15. Reuben, E. and F. van Winden, Fairness perceptions and prosocial emotions in the power to take. J Econ Psych, 2010. 31: p. 908-922.

16. Falk, A., E. Fehr, and U. Fuschbacher, On the nature of fair behavior. Econ Inquiry, 2003. 41(1): p. 20-26.

17. Konow, J., Which is the fairest one of all? A positive analysis of justice theories. J Econ Lit, 2003. 41: p. 1186-1239.

18. Rawls, J., A theory of justice1971, Cambridge: Harvard University Press.

19. Page, K.M. and M.A. Nowak, A generalized adaptive dynamics framework can describe the evolutionary Ultimatum Game. J Theor Biol, 2001. 209(2): p. 173-179.

20. Braun, D.A., P.A. Ortega, and D.M. Wolpert, Nash equilibria in multi-agent motor interactions. PLoS Comput Biol, 2009. 5(8): p. e1000468.

21. Killingback, T. and E. Studer, Spatial Ultimatum Games, collaborations and the evolution of fairness. Proc Biol Sci, 2001. 268(1478): p. 1797-1801.

22. Nowak, M.A., K.M. Page, and K. Sigmund, Fairness versus reason in the ultimatum game. Science, 2000. 289(5485): p. 1773-1775.

23. Page, K.M., M.A. Nowak, and K. Sigmund, The spatial ultimatum game. Proc Biol Sci, 2000. 267(1458): p. 2177-2182.

24. Sigmund, K., C. Hauert, and M.A. Nowak, Reward and punishment. Proc Natl Acad Sci U S A, 2001. 98(19): p. 10757-10762.

25. Duan, W.Q. and H.E. Stanley, Fairness emergence from zero-intelligence agents. Phys Rev E Stat Nonlin Soft Matter Phys, 2010. 81(2 Pt 2): p. 026104.

26. Li, X. and L. Cao, Largest Laplacian eigenvalue predicts the emergence of costly punishment in the evolutionary ultimatum game on networks. Phys Rev E Stat Nonlin Soft Matter Phys, 2009. 80(6 Pt 2): p. 066101.

27. Sánchez, A. and J.A. Cuesta, Altruism may arise from individual selection. J Theor Biol, 2005. 235(2): p. 233-240.

28. Bolton, G.E., A comparative model of bargaining: theory and evidence. Am Econ Rev, 1991. 81: p. 10961136.

29. Tam, D.N., Contributing factors in judgment of fairness by monetary value. BMC Neuroscience, 2011. 12(Suppl 1): p. P329.

30. Tam, D.N., Quantification of fairness bias by a Fairness-Equity Model. BMC Neuroscience, 2011. 12(Suppl 1): p. P327.

31. von Neumann, J., O. Morgenstern, and A. Rubinstein, Theory of games and economic behavior1953, Princeton, NJ: Princeton University Press.

32. Camerer, C., Behavioral game theory: Experiments in strategic interaction2003: Princeton University Press. 
Tam, N. D. (2014). Quantification of Fairness Perception by Including Other-Regarding Concerns Using a Relativistic Fairness-Equity Model. Advances in Social Sciences Research Journal, 1(4), 159-168

33. Kagel, J.H. and A.E. Roth, The handbook of experimental economics1995: PRINCETON University Press.

34. Rilling, J.K., et al., The neural correlates of theory of mind within interpersonal interactions. Neuroimage, 2004. 22(4): p. 1694-1703.

35. Komorita, S.S., Attitude content, intensity, and the neutral point on a Likert scale. J Soc Psychol, 1963. 61: p. 327-334.

36. Tam, N.D., Quantification of happy emotion: Proportionality relationship to gain/loss. Psychol Behav Sci, 2014. 3(2): p. 60-67.

37. Tam, N.D., Quantification of happy emotion: Dependence on decisions. Psychol Behav Sci, 2014. 3(2): p. 68-74.

38. Tam, D.N., Computation in emotional processing: quantitative confirmation of proportionality hypothesis for angry unhappy emotional intensity to perceived loss. Cogn Comput, 2011. 3(2): p. 394-415.

39. Tam, N.D., Quantification of fairness bias in relation to decisions using a relativistic fairness-equity model. Advances in Social Sciences Research Journal, 2014. 1(4): p. 173-182 